\title{
3 Research Square \\ Bleeding Complication in a Patient with \\ Concomitant Use of Rivaroxaban and Saffron Supplement: A Case Report
}

\author{
Maryam Daei \\ Alborz University of Medical Sciences \\ Hossein Khalili \\ Tehran University of Medical Sciences \\ Zinat Heidari ( $\nabla$ heidarizn@mums.ac.ir) \\ Mashhad University of Medical Sciences https://orcid.org/0000-0003-4362-7658
}

\section{Case report}

Keywords: Direct oral anticoagulants, DOACs, Rivaroxaban, Saffron, Crocin, Herb-drug interaction

Posted Date: June 18th, 2021

DOI: https://doi.org/10.21203/rs.3.rs-579040/v1

License: (c) (i) This work is licensed under a Creative Commons Attribution 4.0 International License.

Read Full License 


\section{Abstract \\ Background}

Direct oral anticoagulants (DOACs) carry a lower potential risk of food/herb and drug interactions compared with oral vitamin $\mathrm{K}$ antagonists. However, as a new class of medications some of these interactions have not been fully known.

\section{Case presentation:}

A 64-year old male with a medical history of non-valvular atrial fibrillation presented to the emergency department with a complaint of acute onset epistaxis and bleeding gums following the concomitant use of rivaroxaban and saffron supplement. Rivaroxaban plasma concentration was $54 \mathrm{ng} / \mathrm{ml}$ with a postintake delay of 17 hours. The results of laboratory tests were unremarkable except for platelet function tests. Whole blood multiple electrode aggregometry was performed to assess platelet function. Area under the aggregation curve (AUC) values were 83 and 51 aggregation unit (AU)*min by arachidonic acid and adenosine diphosphate-induced platelet aggregation tests, respectively. As the patient had not taken any antiplatelet medication, platelet dysfunction was greatly attributed to the saffron supplement. The patient was immediately admitted to hospital and received local hemostatic measures and tranexamic acid. Moreover, saffron was discontinued permanently and rivaroxaban was paused for 24 hours. The bleeding stopped a few hours later and the patient was discharged after 2 days in a good general condition. Subsequently, he was followed up at 4, 8, and 12-week intervals. He was in a stable clinical condition with no bleeding complications. The patient was advised to consult with his doctor or pharmacist before taking any supplement or herbal medicine to ensure possible interactions.

\section{Conclusions}

It seems that coadministration of DOACs and saffron supplements should be avoided due to the potential drug-herbal interactions and possible risk of subsequent bleeding complications. However, further studies are needed to confirm the findings and assess the clinical significance.

\section{Background}

Direct oral anticoagulants (DOACs) as a relatively new class of medications have received Food and Drug Administration (FDA) approval for the treatment and/or prevention of arterial and venous thromboembolic events since 2010. The favorable pharmacokinetic and pharmacodynamic characteristics of DOACs make them the preferred anticoagulants in most approved indications. They carry a lower potential risk of food/herb and drug interactions compared with warfarin [1]. However, as a new class of medications some of these interactions have not been fully known. 
Saffron (Crocus sativus L.), as a well-known precious spice, has been used for centuries. Previous studies have investigated the protective effects of saffron in the treatment and/or prevention of cardiovascular, nervous system, psychologic, metabolic, infertility, menstruation, and cancer disorders. The main therapeutic properties are attributed to the saffron carotenoids including crocins and crocetin [2].

According to our literature review, it is the first report of saffron-rivaroxaban interaction which led to minor bleeding in a patient.

\section{Case Presentation}

A 64-year old male with a medical history of non-valvular atrial fibrillation (NVAF) for 2 years, arthritis rheumatoid and hypercholesterolemia for 4 years presented to the emergency department with a complaint of acute onset epistaxis and bleeding gums for the first time. Regular medications included rivaroxaban (20 mg once daily), methotrexate (20 mg once weekly), prednisolone ( $5 \mathrm{mg}$ once daily), folic acid (1 mg once daily), and atorvastatin (40 mg once daily). He noted that the last dose of rivaroxaban was on the previous evening. No new medications had been recently received. He denied alcohol consumption. After taking a detailed medical history, he mentioned the recent use of Krocina ${ }^{\circledR}$ (an herbal supplement tablet containing $15 \mathrm{mg}$ of crocin) twice daily for a 2-week period. He had started taking Krocina ${ }^{\circledR}$ due to feeling of sadness without consulting a physician or pharmacist.

Patient's vital signs were stable at the time of admission. The results of physical examination were unremarkable except for continuous severe epistaxis and bleeding gums. Other possible organic causes of bleeding were excluded following ENT and periodontal consultation. The laboratory tests were within the normal or acceptable range: WBC $10,400 / \mathrm{mm}^{3}[4,500-11,000]$, hemoglobin $14.1 \mathrm{~g} / \mathrm{dL}$ [13.5-17.5], hematocrit $40 \%$ [38.3-48.6\%], platelet count $218,000 / \mathrm{mm}^{3}[150,000-450,000]$, anti-factor Xa activity 0.8 $\mathrm{IU} / \mathrm{mL}$ [0.6-1.0], INR 1 [0.8-1.1], aPPT $40 \mathrm{sec}$ [30-40], serum creatinine $0.9 \mathrm{mg} / \mathrm{dL}$ [0.74-1.35], bilirubin total/direct 0.5/0.1 mg/dL [<1.2/<0.4], alkaline phosphatase $125 \mathrm{IU} / \mathrm{L}$ [20-140], AST $33 \mathrm{U} / \mathrm{L}[<40]$, ALT $24 \mathrm{U} / \mathrm{L}[<56]$, and albumin $3.3 \mathrm{~g} / \mathrm{dL}$ [3.4-5.4]. The HAS-BLED Score was 0 point.

Rivaroxaban plasma concentration was $54 \mathrm{ng} / \mathrm{ml}$ [expected trough level: 6-137] with a post-intake delay of 17 hours. Whole blood multiple electrode aggregometry (MEA), as an established valid assay, was performed to assess platelet function [3]. Area under the aggregation curve (AUC) values were 83 and 51 aggregation unit $(A U){ }^{\star}$ min by arachidonic acid (AA) and adenosine diphosphate (ADP)-induced platelet aggregation tests (ASPI and ADP), respectively. Antiplatelet drugs decrease platelet aggregation level by an AUC value of lower than $300 \mathrm{AU} * \min [4]$.

The patient was immediately admitted to hospital and received local hemostatic measures (including cold compress and mechanical pressure) and tranexamic acid (10 mg/ $\mathrm{kg}$ as an intravenous bolus followed by oral dose of 1 gram every 12 hours for 48 hours). Moreover, Krocina ${ }^{\circledR}$ was discontinued permanently and rivaroxaban was paused for 24 hours. The bleeding stopped a few hours later and the patient was discharged after 2 days in a good general condition. Rivaroxaban was restarted due to the 
high thrombotic risk of AF. The patient was advised to consult with his doctor or pharmacist before taking any supplement or herbal medicine to ensure possible interactions. After discharge, the patient was followed up at 4, 8, and 12-week intervals. He was in a stable clinical condition with no bleeding complications.

\section{Discussion}

To the best of our knowledge, we report here the first case of crocin-rivaroxaban interaction that led to epistaxis and bleeding gums in a patient. He had arbitrarily started daily use of crocin since two weeks before the onset of bleeding. To identify the underlying cause of bleeding, the laboratory tests were done. Interestingly, the results were unremarkable except for platelet function tests. His platelet dysfunction was comparable to aspirin and/or clopidogrel $[5,6]$. As the patient had not taken any antiplatelet medication, platelet dysfunction was greatly attributed to the crocin.

Possible antiaggregatory effects of saffron were studied on human platelets. Saffron supplementation inhibited platelet aggregation induced by ADP, epinephrine, and collagen in a dose-dependent manner. Moreover, it may interfere with the regulation of calcium influx and signaling pathway in activated platelets. Additionally, a same binding site for aspirin and saffron carotenoids in cyclooxygenase I enzyme has been shown in silico studies [7-9].

Although saffron is considered a safe natural product with unrecognized drug interactions, but caution should be taken in concomitant use with anticoagulant, antiplatelet, and antihypertensive medications [10].

Rivaroxaban is the major substrate of CYP3A4 enzyme and P-glycoprotein (P-gp) efflux pump. As a result, its concurrent systemic treatment with strong inhibitors or inducers of CYP3A4 and P-gp should be avoided due to the safety and efficacy concerns. Moreover, pharmacodynamic interactions of rivaroxaban with antiplatelet medications, other anticoagulants, and non-steroidal anti-inflammatory drugs (NSAIDs) should be considered. In addition, there are recognized rivaroxaban-herbal interactions via the pharmacokinetic and/or pharmacodynamic pathways that lead to therapeutic failure or toxicities. Garlic, ginger, ginkgo biloba, ginseng, green tea, and horse chestnut supplements have been known with anticoagulation or antiplatelet activities and caution is needed in concomitant use with rivaroxaban [11].

Based on this experience, saffron appears to have a pharmacodynamic interaction with rivaroxaban. However, further studies are needed to clarify the precise mechanism.

\section{Conclusions}

It seems that coadministration of DOACs and saffron supplements should be avoided due to the potential drug-herbal interactions and possible risk of subsequent bleeding complications. However, further studies are needed to confirm the findings and assess the clinical significance. 


\section{Abbreviations}

DOACs: Direct oral anticoagulants

FDA: Food and Drug Administration

NVAF: Non-valvular atrial fibrillation

MEA: Multiple electrode aggregometry

AUC: Area under the aggregation curve

AU: Aggregation unit

AA: Arachidonic acid

ASPI-test: Arachidonic acid induced platelet aggregation test

ADP: Adenosine diphosphate

ADP-test: Adenosine diphosphate induced platelet aggregation test

P-gp: P-glycoprotein

NSAIDs: Non-steroidal anti-inflammatory drugs

\section{Declarations}

Ethics approval and Consent to participate: In Iran, a case report does not require ethics approval. Our study adhered to the Ethical Guidelines for Medical and Health Research Involving Human Subjects established by Ministry of Health and Medical Education of Iran.

Consent for publication: Written informed consent was obtained from the patient for publication of this case report.

Availability of data and material: The datasets obtained and analyzed in the current study are available from the corresponding author on reasonable request.

Competing interests: The authors declare that they have no competing interests.

Funding: The authors did not receive support from any organization for the submitted work.

Authors' contributions: Zinat Heidari and Maryam Daei treated the patient and wrote the manuscript. Hossein Khalili revised and edited the manuscript. All authors read and approved the final manuscript.

Acknowledgements: Not applicable. 


\section{References}

1. Chan N, Sobieraj-Teague M, Eikelboom JW. Direct oral anticoagulants: evidence and unresolved issues. Lancet. 2020;396(10264):1767-76.

2. Srivastava R, Ahmed H, Dixit RK, Dharamveer, Saraf SA. Crocus sativus L.: A comprehensive review. Pharmacogn Rev. 2010;4(8):200-8.

3. Harrison P. Platelet function testing. In: Post TW,editor. UpToDate. Waltham, MA; 2019.

4. Jámbor C, Weber CF, Gerhardt K, Dietrich W, Spannagl M, Heindl B, et al. Whole blood multiple electrode aggregometry is a reliable point-of-care test of aspirin-induced platelet dysfunction. Anesthesia and analgesia. Anesth Analg. 2009;109(1):25-31.

5. Abdullah WZ, Bakar SA, Zain WSWM, Yusof Z, Mustaffa R, Hassan R. Aspirin Effects on Platelets Using Whole Blood Tested by Platelet Aggregometry: A Comparative Study for Test Validation in a Clinical Hemostasis Laboratory. Laboratory Medicine. 2013;44(1):90-6.

6. Zhang HZ, Yu LH, Kim MH. Effect of different anticoagulants on multiple electrode platelet aggregometry after clopidogrel and aspirin administration in patients undergoing coronary stent implantation: a comparison between citrate and hirudin. Platelets. 2013;24(5):339-47.

7. Jessie SW, Krishnakantha TP. Inhibition of human platelet aggregation and membrane lipid peroxidation by food spice, saffron. Mol Cell Biochem. 2005;278(1-2):59-63.

8. Thushara RM, Hemshekhar M, Santhosh MS, Jnaneshwari S, Nayaka SC, Naveen S, et al. Crocin, a dietary additive protects platelets from oxidative stress-induced apoptosis and inhibits platelet aggregation. Mol Cell Biochem. 2013;373(1-2):73-83.

9. Sinakosa ZM, Geromichalosb GD. The effect of saffron (Crocus sativus) carotenoids on hemostasis and atherosclerosis. Next Generat Sequenc Applic. 2016;3:127.

10. Modaghegh MH, Shahabian M, Esmaeili HA, Rajbai O, Hosseinzadeh H. Safety evaluation of saffron (Crocus sativus) tablets in healthy volunteers. Phytomedicine. 2008;15(12):1032-7.

11. Steffel J, Collins R, Antz M, Cornu P, Desteghe L, Haeusler KG, et al. 2021 European Heart Rhythm Association Practical Guide on the Use of Non-Vitamin K Antagonist Oral Anticoagulants in Patients with Atrial Fibrillation. Europace. 2021. 